\title{
Biological effect on blood cerebrospinal fluid barrier due to radio frequency electromagnetic fields exposure of the rat brain in vivo
}

\author{
A. Ushiyama $\cdot$ H. Masuda $\cdot$ S. Hirota $\cdot$ K. Wake $\cdot$ \\ H. Kawai $\cdot$ S. Watanabe $\cdot$ M. Taki $\cdot$ C. Ohkubo
}

Published online: 1 July 2007

(C) Springer Science+Business Media, LLC 2007

\begin{abstract}
Concerns on health effects of radiofrequency (RF) signals have been discussed. Particularly, the effect on the central nerve system is one of main interest among the general public. So far, there are lots of studies regarding the RF effect on the Blood Brain Barrier (BBB), but no study of the RF effect on the Blood Cerebrospinal fluid Barrier (BCB). In this study we explored the effect on BCB function by radiofrequency electromagnetic fields (RFEMF) exposure. To attain this purpose, we set about to make a real-time measuring system for $\mathrm{BCB}$ function using a micro-perfusion method and examined the short time exposure experiment using rats. Our data suggested the 30 min single exposure of $1.5 \mathrm{GHz}$ RF-EMF at the brain average SARs of $9.5 \mathrm{~W} / \mathrm{kg}$ for adult and $10.4 \mathrm{~W} / \mathrm{kg}$ for juvenile, did not affect $\mathrm{BCB}$ function in rats under the experimental conditions.
\end{abstract}

Keywords Cellular phone - Radio-frequency electromagnetic field · Blood cerebrospinal barrier . Rat

A. Ushiyama $(\bowtie) \cdot$ H. Masuda $\cdot$ S. Hirota

Department of Environmental Health, National Institute of Public Health, 2-3-6 Minami, Wako, Saitama 351-0197, Japan e-mail: ushiyama@niph.go.jp

K. Wake $\cdot$ H. Kawai $\cdot$ S. Watanabe

EMC Group, Applied Electromagnetic Research Center, Research Department 3, National Institute of Information and Communications Technology, Tokyo, Japan

\section{Taki}

Department of Electrical and Electronic Engineering, Tokyo Metropolitan University, Tokyo, Japan

C. Ohkubo

Radiation and Environmental Health, World Health

Organization, Geneva, Switzerland

\section{Introduction}

Possible health effects of weak RF-EMF have been discussed worldwide. Particularly, the effect on the central nerve system is one of main interest among the general public (D'Andrea et al. 2003; Sienkiewicz et al. 2005). However, further scientific evidences are needed for better health risk assessment, particularly; the effect on the bloodbrain barrier (BBB) is still controversial issue. The BBB prevents large molecules in the blood from getting into the brain. This barrier protects the brain from foreign toxic substances. A number of causes, such as edema, anoxia, hypertension, and ionizing radiation, have been shown to induce the BBB changes, often increasing permeability of substances to the brain. Several studies indicate the BBB permeability increase by RF-EMF exposure (Salford et al. 1994, 2003; Persson et al. 1997), but in many replication or follow-up studies most results were negative (Tsurita et al. 2000; Finnie et al. 2001, 2002). Overall, the BBB leakage studies report both positive and negative findings. At high SAR levels, the effects are consistent with local temperature elevations in the brain and increased blood flow. However, at lower SARs, changes in BBB function and permeability are very difficult to detect, if RF-EMF triggers some effects. On the other hand, to ensure the safety of using RF-EMF, the evaluation from the several aspects of the brain function must be considered. From an anatomical point of view, it is known that the brain barrier consist of two kinds of barrier, the BBB and the Blood Cerebrospinal fluid Barrier (BCB) (Bradbury 1972). Physiologically, $\mathrm{BCB}$ makes the chemical environment of the cerebrospinal fluid stable, and provides a protective role for the brain. Although lots of studies regarding the RF effect on the BBB have been done as described above, there is no study of the RF effect on the BCB function. Therefore we 
focused on the effect on the BCB function by RF-EMF exposure.

In this study, we developed a real-time measuring system for the BCB function which defined same as the BBB in a broad sense. We employed micro-perfusion method which optimized to collect the cerebrospinal fluid (CSF) from the rat brain in vivo. By using this method, we performed the short time exposure of the $1.5 \mathrm{GHz}$ RF-EMF to the rat brain, and monitored albumin leakage from the blood to CSF, which reflects the BCB function.

\section{Materials and methods}

\subsection{Animals and surgical procedure}

Experiments were performed on male Sprague-Dawley rats (Tokyo Laboratory Animals Science Co., Ltd., Tokyo, Japan). Rats were individually housed in cages with laboratory chow (FR-2, Funabashi Farms Co., Ltd., Chiba, Japan) and tap water ad libitum. The animal room was adjusted to 12/12 h light/dark cycle automatically. The ambient temperature and the relative humidity were maintained at $23.0 \pm 1.0^{\circ} \mathrm{C}$ and $50 \pm 5 \%$, respectively.

All surgical procedures were performed under sterile conditions. Anesthesia was initiated with a cocktail of ketamine and xylazine (9:1), at the concentration of $100 \mathrm{mg} / \mathrm{kg}$ by IM injection. Rats were set on a stereotaxic instrument (SR-5N; Narishige Scientific Instrument Lab., Tokyo, Japan), and an incision was made in the rat's scalp to expose coronal sutures. A hole was made at $0.8 \mathrm{~mm}$ caudal and $1.3 \mathrm{~mm}$ lateral to the sagittal suture by a drill machine (UC200; Urawa Co., Saitama, Japan). A guide cannula (NG-8FS; Eicom Co., Kyoto, Japan) was introduced to one of the lateral ventricles through the hole and secured to the skull with acrylic dental cement. At the end of experiment, Evans blue dye was injected through the cannula into the lateral ventricle to confirm the placement of the cannula.

\subsection{Experimental protocol}

After $48 \mathrm{~h}$ since surgery, the rats were again anesthetized, and a push pull cannula (NDP-I-8-01FEP; Eicom Co.) was inserted into a guide cannula. The inlet and the outlet of the cannula were connected to the push-pull micro perfusion pump unit (EP-70, Eicom Co.) which enabled the collection of CSF continuously. At the beginning of RF-EMF exposure, a bolus of artificial CSF containing fluorescein isothiocyanate labeled albumin (FITC-albumin, $20 \mathrm{mg} / \mathrm{kg}$ bodyweight; Sigma-Aldrich Inc., St. Louis, MO, U.S.A.) injected into caudal vein and CSF perfusion was started at a rate of $1 \mu \mathrm{l} / \mathrm{min}$. We used " 8 " shaped loop antenna for
RF-EMF exposure and prepared 3 exposure groups and sham exposure for adult and juvenile rats, described below. In this paper, we set the duration of exposure to $30 \mathrm{~min}$ and the cerebrospinal fluid was collected and monitored for a total of $180 \mathrm{~min}$ including the RF-EMF exposure for $30 \mathrm{~min}$. Fluorescent intensity of FITC-albumin in perfusate was monitored by using spectrofluorometry (FP-6500, Jasco Co., Tokyo, Japan) under the condition of excitation / emission wave length at 490/515 nm. Experimental setup was summarized in Fig. 1.

\subsection{Exposure conditions}

For RF exposure, we used " 8 " shaped antenna which we originally developed (Tanaka et al. 2006), and which emulate the condition of RF-EMF exposure emitted cellular phone devise. Rat's head was locally exposed to 1.5 GHz electromagnetic near-field TDMA (time division multiple access) signal for PDC (Personal Digital Cellular, Japanese cellular phone standard) systems by an " 8 ", shaped loop antenna placed $5 \mathrm{~mm}$ upward to the parietal region. RF-EMF intensities were maintained at a brain average Specific Absorption Rate (SAR) of 0.5, 2.0, $9.5 \mathrm{~W} / \mathrm{kg}$ for adult rats and $0.6,2.2,10.4 \mathrm{~W} / \mathrm{kg}$ for juvenile rats, respectively.

\subsection{Ethical approval}

This study was approved by the Animal Care and Use Committee in the National Institute of Public Health, Japan.

\section{Results}

In this study, we developed a new system, which makes it possible to quantify BCB function in vivo with high sensitivity, and can be applied during exposure. The fluorescence

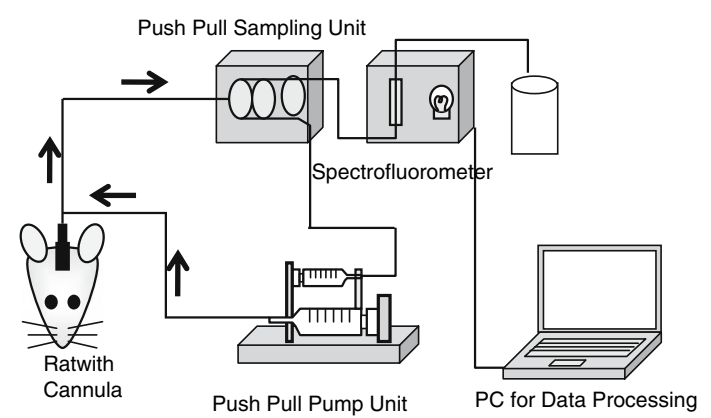

Fig. 1 Schematic diagram of developed system for real-time monitoring of blood barrier function. Artificial CSF flows into the lateral ventricle via inlet of cannula and perfusate comes out via outlet of cannula. Arrows mean the direction of flow 
intensity observed by this system was purely dependent on the concentration of FITC-albumin in perfusate.

Prior to in vivo experiment, we examined an experiment whether our system detect the FITC-albumin in artificial CSF with dose dependent manner (Fig. 2). This data indicate, fluorescence intensity reflects the concentration of FITC-albumin and the sensitivity is extremely high.

To determine the effect of RF-EMF exposure to the rat's head, fluorescent intensity of collected CSF was quantified. As shown in Figures, there is no significant effect among each group in adult (Fig. 3) and juvenile rats (Fig. 4). The data for lower SAR conditions $(0.5,2.0 \mathrm{~W} / \mathrm{kg}$ for adult rats and $0.6,2.2 \mathrm{~W} / \mathrm{kg}$ for juvenile rats were not shown because no statistical difference was detected.

\section{Discussion}

Recently, number of cell phone users is increasing worldwide. Health effects by RF exposure are concerned issue in the general public. Particularly, biological effect on the BBB function by RF exposure is not clear due to variety of the experimental conditions. On the other hand, it is known that the brain barrier consists of two kinds of barrier, BBB and the BCB. So far, there are lots of studies regarding the RF effect on BBB, but no study of the RF effect on the BCB.

From the physiological point of view, the CSF helps in maintaining the chemical environment of the central nervous system and provide a protective role for the brain. To attain these purpose, blood delivered proteins in the CSF is significantly lower than in blood due to the BCB. For example, one of the major proteins of serum, Albumin concentration in the CSF is 200 times lower than that in serum. Under the physiological conditions, this ratio is constant, however once the pathological change is triggered, like cerebrospinal meningitis, CNS injury,

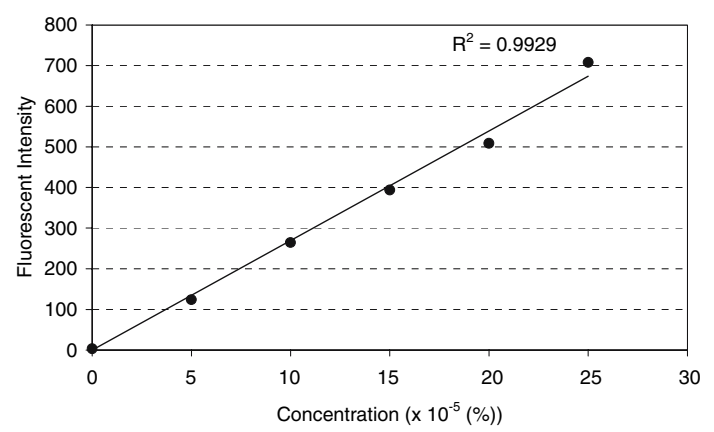

Fig. 2 The relationship between fluorescence intensity, and the FITC-albumin concentration. These two parameters showed linear correlation. From this result, the sensitivity for FITC-albumin of this system was estimated less than $1 \mathrm{ng} / \mathrm{ml}$ in CSF

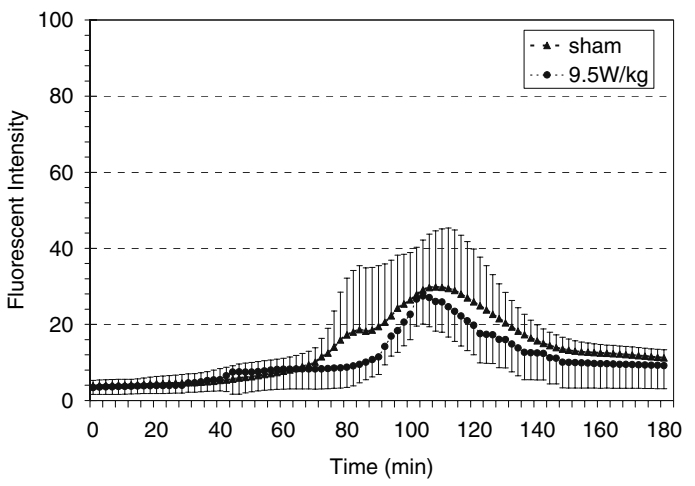

Fig. 3 Fluorescent profiles of "ADULT" rats for sham exposure and RF-EMF exposure. Fluorescence intensity of the collected CSF at each time point was shown. Each plot indicate mean \pm standard error in Sham $(\boldsymbol{\Lambda}, n=6)$ and $9.5 \mathrm{~W} / \mathrm{kg}$ exposure group $(\boldsymbol{\bullet}, n=5)$

Alzheimer disease, this ratio is altered (Raedler and Wiedemann 2006). Therefore, if we can monitor the albumin concentration in the CSF, it must be useful to predict the effect on the BCB by RF-EMF exposure. Since the CSF flows is one way from the lateral ventricles, around the spinal cord, into the sub-arachnoid space and into the venous sinuses, the CSF is produced in the lateral ventricles. Several cannula implantation methods were proposed to collect the CSF from the lateral ventricle. For example, the intraventicular cannula method (one-way cannula) was used for radio-immuno assays (Sanvitto et al. 1987), and push pull cannula method (two-way cannula) was used to determine the CSF production rate in the conscious rats (Baudrie et al. 1990). In this study, we developed a method based on micro perfusion using a push pull cannula which made by non-metal material to prevent interference of RF-EMF. This method enables us to quantify the BCB function in vivo with high sensitivity, and can be applied during RF-EMF exposure. Using this method, we monitored the fluorescence intensity in the

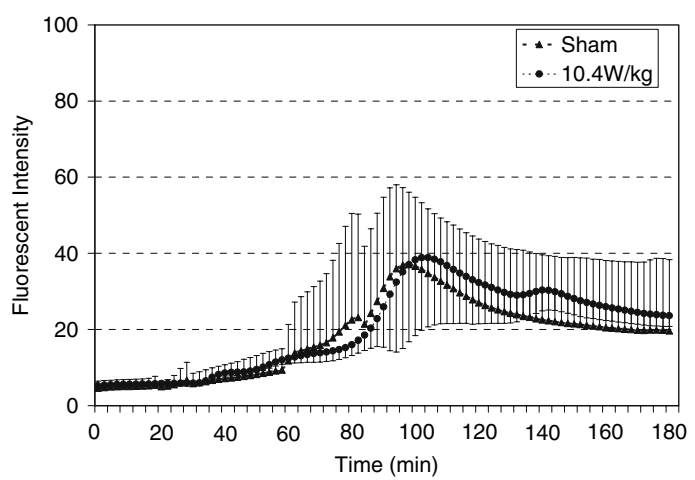

Fig. 4 Fluorescent profiles of "JUVENILE" rats for sham exposure and RF-EMF exposure. Fluorescence intensity of the collected CSF at each time point was shown. Each plot indicate mean \pm standard error in Sham $(\boldsymbol{\Lambda}, n=5)$ and $10.4 \mathrm{~W} / \mathrm{kg}$ exposure group $(\boldsymbol{\bullet}, n=6)$ 
collected CSF, which means the leakage of albumin from blood to CSF.

As shown in this study, after $1.5 \mathrm{GHz}$ RF-EMF exposure for $30 \mathrm{~min}$ using an " 8 " shaped loop antenna, the fluorescence intensity in collected fluid did not increase indicating the BCB did not affected by RF exposure. Same results were observed in both adult and juvenile rats, although it is hypothesized that the juvenile is tend to be more sensitive than adult. As a conclusion, acute and single exposure of $1.5 \mathrm{GHz}$ RF-EMF to the head under this experimental condition does not affect the blood-cerebrospinal fluid barrier function in both adult and juvenile rats. These results may contribute to the evidence for supporting the safety guideline for use of cell phone among children.

Acknowledgement Authors thank to the financial support from the Ministry of Health, Labour and Welfare, Japan.

\section{References}

Baudrie, V., Roullet, J. B., Goureau, Y., Chaouloff, F., \& Elghozi, J. L. (1990). Determination of cerebrospinal fluid production rate using a push-pull perfusion procedure in the conscious rat. Fundam Clin Pharmacol, 4, 269-274.

Bradbury, M. W. B. (1972). Electrolyte disorders and the brain. In Clinical Discoders of Fluid and Electrolyte Metabolism (pp. 1023-1042). New York, London: McGraw-Hill.

D'Andrea, J. A., Chou, C. K., Johnston, S. A., Adair, E. R.: 2003, 'Microwave effects on the nervous system', Bioelectromagnetics Suppl, S107-S147.

Finnie, J. W., Blumbergs, P. C., Manavis, J., Utteridge, T. D., Gebski, V., Swift, J. G., Vernon-Roberts, B., \& Kuchel, T. R. (2001).
Effect of global system for mobile communication (gsm)-like radiofrequency fields on vascular permeability in mouse brain. Pathology, 33, 338-340.

Finnie, J. W., Blumbergs, P. C., Manavis, J., Utteridge, T. D., Gebski, V., Davies, R. A., Vernon-Roberts, B., \& Kuchel, T. R. (2002). Effect of long-term mobile communication microwave exposure on vascular permeability in mouse brain Pathology, 34, 344347.

Persson, B. R., Salford, L. G., \& Brun, A. (1997). Blood-brain barrier permeability in rats exposed to electromagnetic fields used in wireless communication Wireless Network, 3, 455-461.

Raedler, T. J., \& Wiedemann, K. (2006). CSF-studies in neuropsychiatric disorders. Neuro Endocrinol Lett, 27, 297-305.

Salford, L. G., Brun, A., Sturesson, K., Eberhardt, J. L., \& Persson, B. R. (1994). 'Permeability of the blood-brain barrier induced by $915 \mathrm{MHz}$ electromagnetic radiation, continuous wave and modulated at 8, 16, 50, and $200 \mathrm{~Hz}$ Microsc Res Tech, 27, 535-542.

Salford, L. G., Brun, A. E., Eberhardt, J. L., Malmgren, L., \& Persson, B. R. (2003). Nerve cell damage in mammalian brain after exposure to microwaves from GSM mobile phones Environ Health Perspect, 111, 881-883.

Sanvitto, G. L., Azambuja, N. A., \& Marques, M. (1987). A technique for collecting cerebrospinal fluid using an intraventricular cannula in rats. Physiol Behav, 41, 523-524.

Sienkiewicz, Z., Jones, N., \& Bottomley, A. (2005). Neurobehavioural effects of electromagnetic fields. Bioelectromagnetics, Suppl 7, S116-S126.

Tanaka, S., Uno, T., Wake, K., Kawai, H., Watanabe, S., Masuda, H., Ushiyama, A., \& Taki, M. (2006). SAR calculation in immature rats exposed by an 8-shaped loop antenna in $1.5 \mathrm{GHz}$ band. In proceedings of progress In electromagnetics research symposium 2006(pp. 550 , 2-5 August 2006). Tokyo, Japan.

Tsurita, G., Nagawa, H., Ueno, S., Watanabe, S., \& Taki, M. (2000). Biological and morphological effects on the brain after exposure of rats to a $1439 \mathrm{MHz}$ TDMA field. Bioelectromagnetics, 21, 364-371. 